\title{
TTR
}

Traduction, terminologie, rédaction

\section{Saint-Denys Garneau dans le prisme de ses traducteurs hispano-américains}

\section{Madeleine Stratford}

Volume 28, numéro 1-2, 1er semestre-2e semestre 2015

La traduction littéraire et le Canada

Literary translation and Canada

URI : https://id.erudit.org/iderudit/1041655ar

DOI : https://doi.org/10.7202/1041655ar

Aller au sommaire du numéro

\section{Éditeur(s)}

Association canadienne de traductologie

ISSN

0835-8443 (imprimé)

1708-2188 (numérique)

Découvrir la revue

Citer cet article

Stratford, M. (2015). Saint-Denys Garneau dans le prisme de ses traducteurs hispano-américains. TTR, 28(1-2), 153-179. https://doi.org/10.7202/1041655ar
Résumé de l'article

Des quatre " grands aînés » de sa génération (les autres étant Alain Grandbois, Anne Hébert, et Rina Lasnier), le poète québécois Hector de Saint-Denys Garneau est de loin le plus traduit en espagnol. Les premières versions circulent en Amérique latine dès les années 1990. Au cours des années 2000, deux recueils bilingues consacrés à son oeuvre paraissent, traduits par Luis Vicente de Aguinaga. De tous les poèmes traduits, deux l'ont été à quatre reprises : "Accompagnement » et « Cage d'oiseau ». Nous nous intéressons ici à la façon dont le vers libre du poète a été reproduit. Nous déterminerons sous quel angle les traducteurs ont abordé la forme des poèmes pour évaluer, s'il y a lieu, la fréquence des combinaisons traductives. Notre analyse permettra de constater comment le vers libre de Saint-Denys Garneau est donné à lire au public hispano-américain et à quel point son style correspond aux originaux. 


\title{
Saint-Denys Garneau dans le prisme de ses traducteurs hispano-américains
}

\author{
Madeleine Stratford \\ Université du Québec en Outaouais
}

\begin{abstract}
Résumé
Des quatre «grands aînés» de sa génération (les autres étant Alain Grandbois, Anne Hébert, et Rina Lasnier), le poète québécois Hector de Saint-Denys Garneau est de loin le plus traduit en espagnol. Les premières versions circulent en Amérique latine dès les années 1990. Au cours des années 2000, deux recueils bilingues consacrés à son œuvre paraissent, traduits par Luis Vicente de Aguinaga. De tous les poèmes traduits, deux l'ont été à quatre reprises: "Accompagnement» et "Cage d'oiseau ». Nous nous intéressons ici à la façon dont le vers libre du poète a été reproduit. Nous déterminerons sous quel angle les traducteurs ont abordé la forme des poèmes pour évaluer, s'il y a lieu, la fréquence des combinaisons traductives. Notre analyse permettra de constater comment le vers libre de Saint-Denys Garneau est donné à lire au public hispano-américain et à quel point son style correspond aux originaux.
\end{abstract}

Mots-clés: Hector de Saint-Denys Garneau, poésie québécoise, vers libre, traduction poétique, Amérique latine

\section{Abstract}

Out of the four so-called "elders" of his generation (the others being Alain Grandbois, Anne Hébert and Rina Lasnier), Quebec poet Hector de Saint-Denys Garneau is by far the most translated into Spanish. The first versions come out in Latin America as early as in the 1990s. In the 2000s, two bilingual poetry books are published, both of them translated by Luis Vicente de Aguinaga. Among all those Spanish versions, two poems were translated four times: "Accompagnement" and "Cage d'oiseau". We are interested in the way the author's free verse was reproduced. We study how the translators treated those poems' form in order to establish, if need be, the frequency of certain combinations of translation strategies. This analysis will show in what way Saint-Denys Garneau's free verse was presented to the Hispanic American readership, and to what extent these versions correspond to the poet's style in the original French.

Keywords: Hector de Saint-Denys Garneau, Quebec poetry, free verse, poetry translation, Latin America 
Hector de Saint-Denys Garneau est un des plus grands poètes québécois, reconnu pour avoir inauguré au Québec la poésie moderne (Haeck, 1973, p. 95; Mainguy, 2012,p. 32) et qui se serait même hissé au statut de «mythe»(Major, 1972, p. 77-78). En effet, si le vers libre de Saint-Denys Garneau détonnait à la fin des années 1930, alors que la poésie canadienne-française était encore fortement ancrée dans les modèles traditionnels français, celuici a fini par faire école. On dit souvent de Saint-Denys Garneau qu'il écrit dans une langue "vive», "parlée», "dépouillée» mais «intense» (Blais, 1984, p. 29-30; Jones, 2004, p. 13; Nepveu, 1984, p. 20-21), dont la syntaxe transgresse par moments les normes grammaticales (Blais, 1984, p. 30; Melançon, 2004, p. 52). Encore aujourd'hui, plusieurs études sont consacrées à son œuvre. En fait notamment foi le numéro spécial que la revue Études françaises a produit à son sujet en 2012 (vol. 48, n ${ }^{\circ}$ 2). E. D. Blodgett y remarquait que Saint-Denys Garneau «continue à être un des poètes [québécois] les plus fréquemment traduits en anglais" (2012, p. 114). En Amérique latine aussi, il s'avère le plus traduit en espagnol de sa génération, avec deux recueils individuels et une douzaine de poèmes dans cinq anthologies ${ }^{1}$.

Le présent article vise à tracer un portrait des traductions hispano-américaines de l'œuvre de Saint-Denys Garneau, au moyen d'une étude de cas de deux de ses poèmes les plus connus, qui s'avèrent aussi les plus traduits en espagnol: "Cage d'oiseau» et "Accompagnement». La plus ancienne version d' "Accompagnement», signée Isabel Paraíso de Leal, est parue en 1973 dans la revue vénézuélienne Árbol de fuego. Cette traductrice est la seule du corpus à ne pas avoir traduit aussi "Cage d'oiseau». Les traductions de Sara Cohen sortent en Argentine en 2006, dans un ouvrage hybride entre l'essai et l'anthologie intitulé La frontera de la lengua. Toutes les autres versions sont parues au Mexique: celles de Lorenza Fernández del Valle et Juan Carvajal en 1996 dans l'anthologie Poetas de Quebec, celles de Marco Antonio Campos en 2003 dans l'anthologie Latinos del Norte, et celles de Luis Vicente de Aguinaga dans deux recueils individuels, Pequeña fin del mundo en 2003 (sans "Accompagnement») et Todos y cada uno en 2007 (avec «Accompagnement» et une version révisée de "Cage d'oiseau»). Nous comparerons les cinq versions publiées

1. Pour plus d'information bibliographique, consulter les bases de données TEPOQAL I et II : http://linguistech.ca/tepoqal. 
de chacun des deux poèmes qui serviront de baromètres pour vérifier comment le vers libre de Garneau est donné à lire au public hispano-américain et dans quelle mesure le style des traductions correspond à celui des originaux.

«Cage d'oiseau» et «Accompagnement», qui se suivent l'un l'autre dans Regards et jeux dans l'espace, sont particulièrement représentatifs de l'œuvre de Saint-Denys Garneau sur les plans du contenu et de la forme (Haeck, 1973, p. 97). Plus d'un critique a d'ailleurs tracé des parallèles entre les deux poèmes. Jean-Louis Major, par exemple, juge qu'aucun des poèmes de Saint-Denys Garneau «ne s'accompli[ssent] dans une forme aussi proche» (1972, p.189); Renald Bérubé remarque pour sa part la similarité de leur structure répétitive, où Saint-Denys Garneau «reprend les termes ou les expressions [du premier vers] pour les placer dans des contextes différents, dans des structures syntaxiques ressemblantes, mais un peu dissemblables» (1973, p. 99). Aussi, chaque poème est basé sur une série d'inversions. Dans «Cage d'oiseau », Jacques Blais identifie une structure chiasmatique qui affecte toutes les strophes: «[1]a composition est rigoureusement géométrique : à la verticale, les trois premiers et les trois derniers vers $[\ldots]$ forment un chiasme [...]; à l'horizontale, les vers C D D'C' [strophes 2 et 5] en agencent un deuxième (le troisième chiasme concerne l'assemblage verbal du poème» (1984, p. 46). Dans "Accompagnement", Major avance que les charnières syntaxiques créent un système de contrastes, si bien que «les deux premières strophes s'opposent aux deux dernières» (1972, p. 181). Dans un tel contexte, il n'est pas étonnant que presque tous les traducteurs du corpus aient inclus les deux textes dans leur sélection espagnole, à part Isabelle Paraíso de Leal, qui n'a pas traduit «Cage d'oiseau ».

Antoine Berman a déjà déclaré que la traductologie était «l'équivalent, pour la traduction, du discours critique de la littérature sur elle-même» (1989, p. 676). Comme sœur jumelle de la critique littéraire, la traductologie littéraire peut étudier, par exemple, plusieurs traductions d'un même original dans une seule langue cible donnée. Or, si comparer les œuvres de plusieurs auteurs relève de la littérature comparée, nous proposons que comparer plusieurs traductions d'une même œuvre par différents traducteurs soit vue comme une forme de traductologie comparée. En plus du rapport horizontal traditionnel entre les langues source et cible qui a toujours lieu en traductologie, il en existe un autre, 
vertical celui-là, entre les traductions d'un même texte dans une seule langue cible, ainsi qu'entre les traductions d'un même texte dans une variété de langues cibles. Voilà ce que nous proposons de faire dans la section qui suit, où nous nous pencherons sur la façon dont les traducteurs ont reproduit le vers libre du poète québécois.

Dans un article sur la version anglaise de «Saules» produite par John Glassco, Patricia Godbout s'intéresse «à ce qu'il advient en traduction du rapport entre répétition, contraste et invention" (2009, p. 23). Dans la première partie de l'analyse, Godbout souligne l'importance, dans le cas de Saint-Denys Garneau, de considérer les mots avant tout comme des signes et leur agencement, comme un système ou une architecture. Paraphrasant Barnstone, elle avance même que "traduire le signifié sans se référer à son signifiant équivaut à un meurtre de l'art» (ibid, p. 24). Ces propos invitent à nous demander si l'esthétique de Garneau a survécu dans le prisme des traductions espagnoles de «Cage d'oiseau» et d' «Accompagnement» diffusées en Amérique latine. Pour ce faire, nous relèverons les éléments qui distinguent les différentes versions dans le but de déterminer sous quel angle les traducteurs ont abordé le style garnélien, dans ses manifestations sonores et spatiales (une part du sens, nous le verrons, surgit dans le traitement sonore et spatial des poèmes). Nous classerons nos observations de chacun des aspects en trois catégories: une approche structurelle importera les structures originales, quitte à sacrifier le sens ou à produire un effet d'étrangeté dans la langue cible; une approche fonctionnelle recréera l'effet original à l'aide des ressources propres à la langue cible; une approche adaptative générera un sens ou un effet distinct de l'original ${ }^{2}$.

\section{Aspect sonore}

L'aspect sonore est celui dont parlent le plus les critiques qui s'attardent à la forme des poèmes de Saint-Denys Garneau. Dujka Smoje (1982) consacre même tout un article à leur «musique». S’il y a bel et bien «intrusion de la prose» chez Saint-Denys Garneau, comme l'affirme Pierre Nepveu (1984, p. 18), D. G. Jones rappelle que le poète n'en est pas moins «toujours attentif aux nuances du sens et du son»(2004, p. 13). Marina Zito, qui s'intéresse à l'«architecture» des poèmes de Saint-Denys Garneau, juge que les

2. Pour une discussion détaillée des trois approches de la reproduction de l'aspect sonore, voir Stratford (2011). 
répétitions lexicales et phoniques y sont «étudiées» (2004, p. 109). Pour sa part, Michel Lemaire reconnaît qu'à la première écoute, les poèmes « paraissent agressivement dépourvus de musicalité » (1994, p. 73). Il y distingue bien quelques «suite[s] de vers isométriques» (ibid., p. 82), le plus souvent hepta- ou octosyllabiques, qui ne suffisent cependant pas à créer de régularité au sens strict. Pour Lemaire, le vers libre de Saint-Denys Garneau se définit plutôt "par son opposition même au vers régulier» et c'est «en le dépouillant de ses enjolivements métriques» que l'auteur le rend vraiment poétique (ibid, p. 73). Plutôt que de reposer sur «les réitérations de la versification régulière ", la structure sonore des poèmes puiserait selon lui notamment dans "l'anaphore et la juxtaposition» (ibid, p. 77). Pour ce qui est de la rime, Lemaire explique que SaintDenys Garneau en a fait "systématiquement» usage dans ses poèmes de jeunesse, et ce "pendant plusieurs années", cela sans toujours obéir aux «règles sur leur genre et leur nombre» (ibid., p. 75). Des rimes et assonances occasionnelles persistent d'ailleurs dans plusieurs poèmes de Regards et jeux dans l'espace, dont "Cage d'oiseau» et "Accompagnement». Pour Michael Riffaterre, les éléments formels destinés à remplacer le vers traditionnel doivent être répétés pour que le lecteur puisse les interpréter comme les différentes variables d'une même constante (1983, p. 117). Nous nous attarderons donc ici au type de répétitions qui semblent le plus contribuer à structurer les deux poèmes sur le plan sonore, et qui sont aussi (à première vue, du moins) le plus facilement reproductibles en traduction: les répétitions lexicales (mots ou groupes de mots). Nous verrons qu'en plus de conférer aux poèmes un rythme et un effet de circularité, ces répétitions créent des liens sémantiques entre certains mots et vers clés.

\section{1. "Cage d'oiseau "}

Ce poème, reproduit à l'annexe $\mathrm{I}$, est particulièrement riche sur le plan sonore. C'est le seul du corpus contenant des répétitions des trois types. Sur le plan lexical, les substantifs réitérés sont interreliés métaphoriquement, car ils servent tous à décrire le «je» lyrique: «cage» et "oiseau» d'abord (quatre fois, cinq si l'on compte le titre), suivis de près par «os» (trois fois). La double répétition du substantif «mort», utilisé comme métaphore de l'«oiseau», se rajoute à ce groupe par association sémantique. Ces répétitions des principaux symboles du poème participent selon nous au «rythme haché» que remarque Robert Vigneault (1973, p. 55), en 
particulier parce qu'elles surgissent dans des vers où les sauts de lignes allongent des pauses syntaxiques plutôt qu'ils ne produisent des enjambements. Sur le plan syntaxique, une série de structures se démarque particulièrement: la combinaison «c'» + «est» aux vers 5, 13 et 18, inversée («est-ce») sous la forme d'une anaphore aux vers 16,17 et 18 . Ce réseau met en valeur le désir du sujet de se définir, exprimé dès le premier vers: "Je suis une cage d'oiseau ». La double répétition du mot «avec» (v. 3 et 23), qui apporte une précision tardive aux définitions données, appartiendrait aussi à ce groupe. On pourrait en outre y adjoindre les deux apparitions de «on entend» (v. 7 et 10), qui décrit les actions de l'oiseau et contribue donc aussi à l'élaboration de la définition ${ }^{3}$.

\section{2. "Accompagnement"}

Comme le remarque Vigneault, «les répétitions sont très fréquentes dans ce poème» $(1973$, p. 58), si bien qu'il se révèle un pur bijou d'art combinatoire. Jones, par exemple, relève l'anaphore du syntagme «d'une joie» à la première strophe (2004, p. 13). Mais le poème, reproduit à l'annexe II, compte quatre autres répétitions anaphoriques: «Je marche» (v. 1 et 4 ); «Mais» (v. 6, 10); «Par» (v. 11, 12,14) et «Avec» (v. 17, 18). Ces réitérations, combinées avec la récurrence du pronom «je» (Vigneault, 1973, p. 57), qui revient neuf fois, confèrent au poème un effet de circularité. Celuici est appuyé par un réseau de répétitions de mots pleins concentré dans les deux premières strophes, mais que l'on retrouve aussi, dans une moindre mesure, dans la dernière: le substantif «joie» et le pronom «moi» (six fois chacun); le substantif «pas» (quatre fois, sept si l'on compte l'adverbe de négation); la locution adverbiale «à côté de» (quatre fois), le verbe «marche» (trois fois). Ce réseau de répétitions lexicales vient appuyer la trame du poème annoncée

3. Notons qu'en plus des répétitions lexicales, «Cage d'oiseau» compte bon nombre de répétitions phoniques, voire, comme l'a remarqué Blodgett, «un certain penchant pour la rime» aux strophes 4, 6 et 7 (2012, p. 117) (voir aussi T. D. Ryan, 2003, p. 47). La quatrième strophe, présente une rime en [ku] aux vers 8, 9 et 10, qui est développée par une assonance en écho aux vers 9 et 10 («tout à coup »; « roucoule»). Il s'agit d'une rime particulièrement riche. Non seulement elle est la seule du poème à être constituée de deux phonèmes, mais la concentration du son [u] illustre, sur le plan sonore, le roucoulement qui est décrit dans la strophe. La sixième strophe présente pour sa part une suite de rimes pauvres en [e] (v. 15, 16,18), qui refait surface à la septième strophe (v. 19,20): si ces rimes ne contribuent pas vraiment à la trame symbolique, elles n'en structurent pas moins la musique. 
par le titre, soit la contiguïté (mais non l'identité) de «je» et «joie», dont Major dit qu'elle est "réalisée stylistiquement [...] dans l'action "marcher à côté de» (1972, p. 185). Comme le souligne Philippe Haeck, «la juxtaposition des mots «je», «joie» et «moi» est sans doute heureuse à cause de leur structure phonologique semblable qui donne plus de force à leur combinaison» (1973, p. 100). Comme «moi» et «joie» reviennent trois fois ensemble en fin de vers, plusieurs y ont d'ailleurs vu un schéma rimé (Major, 1972, p. 46). On pourrait voir ce schéma enrichi, à la deuxième strophe, par l'assonance en [wa] produite par «trottoir» (v. 6) et l'écho en [a] de «pas-là» au vers suivant ${ }^{4}$.

\subsection{Traduction espagnole des répétitions lexicales}

Certes, il n'est pas rare que les répétitions lexicales disparaissent en traduction, et ce, même lorsquelles jouent comme ici un rôle de structuration. Selon Nitsa Ben Ari, le fait d'éviter les répétitions serait un trait si répandu qu'il s'agirait même d'un "universel de la traduction» $(1998$, p. 2). Dans les traductions à l'étude (voir les annexes I et II), beaucoup de répétitions lexicales originales sont reportées, mais leur proportion et leur traitement diffèrent selon le poème analysé et aussi selon le type de mots répétés. Dans "Cage d'oiseau», par exemple, les répétitions de substantifs sont systématiquement reproduites, mais on constate une plus grande variation pour les mots appartenant à d'autres catégories grammaticales. En effet, toutes les versions de «Cage d'oiseau " contiennent exactement le même nombre de répétitions des équivalents des mots "cage», "oiseau», "os» et "mort». En revanche, l'effet miroir entre «c'est» et «est-ce» était voué à être atténué, la structure interrogative ne se construisant pas ainsi espagnol. Cela dit, les versions de Fernández del Valle et

4. La troisième strophe présente aussi des assonances en fin de vers qui s'approchent de la rime: l'une en [i] «compagnie» (v. 9) et «alchimie» (v. 11), l'autre en [ã] avec «échanges» (v. 10) et «sang» (v. 12). D’ailleurs, le phonème [ã] est omniprésent dans le poème, à commencer par le titre. Il n'y a pas une strophe qui ne le contienne pas, et sa présence augmente au fil des strophes: une fois dans la $1^{\text {re }}$ strophe, trois fois dans la $2^{\mathrm{e}}$, six fois dans la $3^{\mathrm{e}}$ et sept dans la $4^{\mathrm{e}}$. En outre, le parallèle sonore entre «compagnie» et «alchimie » est intéressant en ceci que le poète semble incapable d'élucider le mystère du concept d'«Accompagnement» évoqué par le titre. Pour sa part, l'assonance entre «échanges» et «sang» accentue l'image de «transfusions» exprimée au vers 12 . 
Carvajal et de Aguinaga produisent toutes les deux une structure anaphorique qui rappelle l'originale, les premiers par «Y eres tú / Y soy yo » et le second, par «No serás / No seré». Dans une certaine mesure, Cohen établit aussi un parallèle entre les vers au moyen de l'adverbe "acaso ", mais la structure syntaxique des deux vers diffère trop pour évoquer l'anaphore. Pour sa part, la double répétition du segment «on entend» est presque reprise chez Fernández del Valle et Carvajal et chez Campos, mais avec une variation syntaxique dans les deux cas: «Se oyen / Se oye» chez les uns; «Se oye / Se le oye» chez l'autre. Quant à lui, Aguinaga a gardé le même verbe, mais a opté pour la première personne du pluriel à la quatrième strophe, ce qui annule le parallèle entre les deux segments, d'autant plus que le pronom réflexif «se» a été remplacé l'objet direct «la»: «Se oyen / La oímos». Cohen a elle aussi choisi le «nosotros» pour la deuxième occurrence, et comme elle a changé le verbe, il n'y a plus aucune trace de répétition lexicale.

Dans «Accompagnement», les solutions traductives sont beaucoup plus variées, comme si l'on avait jugé que l'architecture de ce poème ne reposait pas autant sur les répétitions lexicales que celle du poème précédent. Pourtant, nous avons vu plus haut qu' "Accompagnement» se construit lui aussi sur un réseau de répétitions de mots et de groupes de mots, peut-être même encore plus que "Cage d'oiseau». D'abord, tous les traducteurs ont reproduit l'anaphore «d'une joie» (F. del Valle et Carvajal varient un peu en élidant le deuxième «de»), mais le traitement des autres répétitions du mot «joie» diffère. Paraíso de Leal et Cohen ont utilisé le même mot six fois, comme en français («alegría»); les trois autres ont varié en optant à la strophe 2 pour un mot dérivé, qui conserve cependant un aspect itératif: un adjectif chez Campos («gozoso») et chez Fernández del Valle et Carvajal ( «alegre») et un participe présent chez Aguinaga ("gozando»). De même, seuls trois traducteurs ont reproduit l'anaphore de «Je marche » de façon structurelle (Paraíso de Leal, Campos et Cohen). Aguinaga, plus fonctionnel, a troqué la troisième répétition de «camino» pour «andar» et Fernández del Valle et Carvajal, plus adaptatifs, ont carrément utilisé trois verbes différents («Voy»; "Camino»; "marcha»). Les autres mots répétés en début de vers ont tous été repris dans la majorité des traductions, sauf le "par» (strophe 3). Aguinaga a élidé la troisième répétition de (approche fonctionnelle), tandis que Fernández del Valle et Carvajal, plus 
adaptatifs, ont remplacé la triple séquence par une série de mots outils dépourvue de cohésion («para», «por», «en»). Dans le cas du substantif "pas», les traducteurs ont conservé les quatre répétitions, à une exception près: Fernández del Valle et Carvajal ont opté pour l'importation de l'expression française «pas de joie» à la dernière strophe. Ils ont dû voir dans le syntagme «pas de joie » une précision du type de danse évoquée juste avant. Bien que cette interprétation soit défendable, l'emprunt direct reste étrange, car ni l'expression ni le type de danse n'existent vraiment.

Comme on pouvait s'y attendre en espagnol, où le pronom sujet est souvent élidé, presque toutes les répétitions du «je» sujet ont disparu: un seul "yo" se retrouve dans toutes les traductions, mais il traduit « moi », pas «je » (v.7). En fait, la seule version où «yo» réapparaît en position de sujet est celle de Campos (v. 1 et 4). Peutêtre celui-ci a-t-il voulu reporter la structure anaphorique française et créer un effet de circularité. Toutefois, ce pronom sujet semble peu idiomatique en regard des autres conjugaisons de la première personne du poème, toutes à sujet nul. En outre, ces "yo» créent une insistance de type «moi, je» ou (par opposition aux autres) qui était absente en français. Le traitement du pronom «moi» engendre plus de variations, surtout quand il est précédé d' "à côté de ». Cette locution génère en espagnol trois structures équivalentes, qui cohabitent parfois au sein d'une même version: «a lado de mí», «a mi lado» et «al lado mío». Cela dit, le substantif «lado» est présent dans presque tous ces cas, préservant ainsi l'aspect itératif des segments. La seule version plus adaptative à cet égard est celle d'Aguinaga, où «à côté de» est tantôt traduit par «al parejo de» (v. 1), tantôt par «junto a» (v. 4, 5) et tantôt par «a mi lado».

\section{Aspect spatial}

En ce qui a trait à l'aspect spatial des poèmes de Saint-Denys Garneau, il importe de noter que celui-ci nétait pas que poète, mais aussi peintre. Ainsi portait-il une attention particulière à l'apparence de ses textes, en particulier à la ponctuation, qu'il utilise avec parcimonie, à la distribution des vers, qui présentent souvent une indentation, ainsi qu'au découpage des strophes (Blais, 1984, p. 3132; Ryan, 2003, p. 38-39). Zito remarque aussi un usage récurrent de l'italique (2004, p. 109). On pourrait penser que la mise en page relève souvent plus de décisions éditoriales que poétiques, mais Blais rappelle avec pertinence que Saint-Denys Garneau « fut son propre éditeur, qu'il a préparé et surveillé l'impression du recueil» 
(1984,p. 33). En comparant les autographes et les versions publiées, Blais démontre d'ailleurs le caractère intentionnel de l'économie de signes de ponctuation et de la division des strophes, ainsi que de l'ordonnancement des poèmes au sein du recueil (ibid, p. 3133). Or, non seulement les caractéristiques graphiques relevées par les critiques de Garneau se retrouvent dans les originaux à l'étude, mais elles contribuent clairement à leur signification globale. Nous classerons nos observations du traitement de l'espace en quatre catégories, l'une (place des poèmes dans le recueil) correspondant au plan macrotextuel et les trois autres, au plan microtextuel (mise en page, ponctuation et découpe des vers).

\section{1. "Cage d'oiseau "}

Dans l'édition des Poésies complètes de Saint-Denys Garneau de 1949 (qui calque la mise en page de 1937), "Cage d'oiseau» apparaît comme l'avant-dernier poème de Regards et jeux dans l'espace. Le titre est en petites majuscules, aligné à gauche (centré en 1971). Le corps du texte, lui, s'étale sur deux pages, tronqué après la cinquième strophe. Le saut de page se fait exactement au même endroit dans l'édition des Euvres de Garneau en 1971. Or, ce n'est pas là une division innocente: elle arrive en effet juste avant le changement de ton introduit par la sixième strophe, où l'on passe d'un discours affirmatif à un discours interrogatif. Toutefois, les phrases qui constituent cette sixième strophe, bien qu'elles revêtent clairement la structure syntaxique typique de questions, ne portent aucun point d'interrogation. En fait, le seul signe de ponctuation de tout le poème est le point au dernier vers, qui constitue un point final dans tous les sens du terme, marquant visuellement la mort du sujet lyrique évoqué sur le plan thématique: «Il aura mon âme au bec.» Sur le plan des enjambements, les vers sont plutôt courts et leurs indentations ne se situent pas à gauche, comme dans d'autres poèmes de Garneau, mais à droite. Quatre vers particulièrement courts se distinguent visuellement: «Une cage d'oiseau» (v. 2); «Au fond» (v. 11), «Est-ce moi» (v. 17) et «Mon cœur» (v. 21). Là aussi, la mise en page semble entrer en dialogue avec le contenu sémantique du poème. Lus l'un après l'autre, ceux-ci semblent en effet remettre en question la première affirmation du poème: Estce bien moi, cette cage d'oiseau qui contient mon cour (un oiseau), au fond? 


\section{2. "Accompagnement"}

Dans Regards et jeux dans l'espace (comme dans Poésies complètes et Euvres), le poème "Accompagnement» est placé immédiatement après "Cage d'oiseau» et il tient sur une seule page. Si l'on tient compte du fait que Saint-Denys Garneau aurait choisi avec soin l'ordre de ses poèmes, le fait que les deux poèmes se suivent implique qu'ils sont interreliés, si bien qu' «Accompagnement» pourrait constituer une suite, un prolongement de "Cage d'oiseau ». Mais il y a plus: comme il s'agit du tout dernier poème du recueil, que son titre se situe sur une page séparée, écrit en grandes majuscules, et que le corps du texte est en italique, il revêt «le caractère d'un épilogue» (Vigneault, 1973, p. 57). La disposition diffère un peu dans l'édition de 1971, mais elle se démarque toujours en ceci que le titre apparaît en italique audessus du corps du texte (qui, lui, est en lettres romaines). Comme "Cage d'oiseau», "Accompagnement» est très peu ponctué, et la plupart des pauses normalement introduites par des virgules ou des points le sont plutôt par des sauts de lignes, parfois assortis d'un blanc typographique (v. 8, 14, 19 et 21). Le deux-points et les guillemets qui encadraient le $8^{\mathrm{e}}$ vers dans l'autographe ont complètement disparu, ce qui rappelle l'absence de points d'interrogation dans "Cage d'oiseau». Seules deux appositions sautent aux yeux: «des alchimies» (v. 11) et «transposé» (v. 15). Si ces segments n'étaient pas encadrés de virgules, le premier aurait l'air d'un complément du nom "opérations» et le second, d'une épithète du nom «jour». En l'occurrence, le premier ressemble plutôt à un synonyme d' "opérations " servant à en définir la nature, et le second, à une épithète du sujet lyrique, qui renforce l'idée du vers suivant, où il est "porté par la danse». Pour ce qui est des vers, ils sont manifestement plus longs que dans "Cage d'oiseau». Lorsqu'ils sont tronqués, ils ne le sont plus à droite, mais à gauche, faisant visuellement écho au contenu du vers 19: «s'étiolant à ma gauche». Les quatre vers en évidence pourraient à nouveau être lus de façon suivie, cette fois comme un résumé du poème tout entier, où le sujet lyrique affirme que l'«étranger» et lui ne font qu'un: «voilà c'est moi» qui «prend une rue transversale», en passant "par des jeux d'équilibre s'étiolant à ma gauche». Enfin, comme "Cage d'oiseau», "Accompagnement» se termine par un point final qui met une fin définitive à l'action du poème, décrite comme 
un «accompagnement». En effet, un peu comme le sujet lyrique qui voyait son âme emportée dans le bec de l'oiseau, le marcheur voit ici son «pas» disparaître «sous les pieds d'un étranger» qui s'éloigne en prenant une autre rue.

\section{Traductions espagnoles}

\subsection{Place des poèmes dans la sélection traduite}

Toutes les versions espagnoles des deux poèmes font partie d'une sélection; elles ont donc été extirpées du « tout» dont elles faisaient originellement partie. En fait, seules les versions qu'Aguinaga publie en 2007 apparaissent dans un contexte similaire à celui d'origine, comme les deux derniers textes de Regards et jeux dans l'espace. Sur le plan macrotextuel, il s'agit donc de la seule publication que l'on pourrait qualifier de «structurelle», les autres seraient plutôt, à cet égard, fonctionnelles ou adaptatives. Les versions fonctionnelles, par exemple, font un genre de compromis entre la place quoccupaient les poèmes dans le recueil original et celle qu'ils occupent dans la sélection traduite. C'est le cas, par exemple, des traductions de Fernandez del Valle et Carvajal et de Campos, où "Cage d'oiseau» et "Accompagnement» closent la section accordée à Saint-Denys Garneau. Jusqu'à un certain point, c'est aussi un peu le cas de la première sélection traduite par Aguinaga: «Cage d'oiseau » y apparaît en dernière place, un peu de la même façon dont il "clôt» le recueil original, avant l'insertion du genre d'épilogue que constitue «Accompagnement». Pour leur part, les sélections traduites de Cohen et de Paraíso de Leal sont nettement plus adaptatives. D'abord, nous avons vu plus haut que le livre de Cohen est un hybride, "Accompagnement» étant cité dans la section «essai» et «Cage d'oiseau» introduisant plutôt la section anthologique. Dans cet ouvrage, il n'y a donc plus de lien graphique évident entre les deux poèmes. Chez Paraíso de Leal non plus: dans Árbol de fuego, "Accompagnement» précède "Faction». Non seulement il n'est donc plus mis en parallèle avec "Cage d'oiseau», mais il ne joue résolument plus le rôle d'épilogue.

\subsection{Mise en page}

Jusqu'à un certain point, la mise en page de poèmes, peut-être encore plus lorsqu'ils sont inclus dans une anthologie collective, résulte d'un compromis entre les contraintes du format éditorial choisi et la mise en page originale. On pourrait donc dire que 
toutes les versions espagnoles de Saint-Denys Garneau sont, en principe, le résultat d'une approche fonctionnelle. Cela dit, qu'ils aient été faits par le traducteur, le réviseur ou l'éditeur, certains choix de mise en page ont une incidence sur le rythme de lecture du poème, voire sur sa signification globale. Dans le cas de «Cage d'oiseau ", par exemple, toutes les versions s'étendent sur deux pages comme l'original, mais aucune n'est scindée au même endroit que lui (entre les strophes 5 et 6). Fernández del Valle et Carvajal ainsi qu'Aguinaga (2007), par exemple, coupent entre les strophes 6 et 7; Cohen coupe entre les strophes 3 et 4 . On peut considérer ce genre de division comme fonctionnelle, car elle introduit une pause peutêtre plus longue, sans briser pour autant le schéma strophique du poème. En revanche, lorsque le poème est séparé au beau milieu d'une strophe, l'effet produit est d'ordre adaptatif. Ainsi, quand Aguinaga, en 2003, sépare la sixième strophe en deux, il interrompt la suite de questions, qui produisent pourtant une gradation dans le poème. De même, quand Campos coupe la septième strophe après les deux premiers vers, la lecture du poème s'en trouve altérée. Au lieu d'être compris comme une explication de la nature de «todo", «mi corazón / La fuente de la sangre / con la vida dentro» devient tout à coup un agent potentiel de l'action du dernier vers à sujet nul «Tendrá mi alma en el pico». Métaphoriquement parlant, cette interprétation tient la route, puisque la «cage d'os» pourrait être vue comme la «cage thoracique», et l'oiseau, comme le "cœur» qu'elle contient. Cela dit, elle s'éloigne des réseaux de sens établis par la disposition strophique originale.

Par ailleurs, en ce qui a trait à la mise en page globale, notons que la plus structurelle des versions d' «Accompagnement» est sans doute celle d'Aguinaga: le titre apparaît sur une page séparée et le corps du texte est en italique. Cela dit, la version contient un élément adaptatif: le premier vers est en petites majuscules. On pourrait y voir un trait fonctionnel, puisque d'autres premiers vers, ou leur début, sont mis en petites majuscules. Néanmoins, ce formatage semble réservé aux poèmes sans titre. Or, bien que le mot «Accompagnement» soit sur la page précédente, il n'en reste pas moins le titre du poème, du moins aux yeux de l'ensemble de la critique garnélienne. Les petites majuscules induisent donc le lecteur en erreur, d'autant plus que, dans la table des matières, le poème est identifié par son premier vers, le mot «Accompagnement» paraissant plutôt chapeauter une septième 
«section" non numérotée du recueil à laquelle il serait rattaché. Même si cette présentation cadre avec l'interprétation que le poème servirait d'épilogue au recueil, elle produit quand même un effet adaptatif.

\subsection{Ponctuation}

Presque toutes les versions espagnoles, sauf celle de Campos, abordent les signes de ponctuation de façon essentiellement structurelle, reproduisant tout bonnement la distribution originale. La traduction d' «Accompagnement» faite par Cohen se distingue $\mathrm{du}$ lot en ceci que le point final, ainsi que la virgule après «alquimias», ont disparu. Si les signes avaient été retranchés parce que Cohen les avait jugés superflus en fin de vers, celle-ci aurait probablement enlevé la virgule après "traspuesto", ce qu'elle n'a pas fait. En fait, on dirait plutôt des oublis typographiques plus que des choix délibérés: après tout, les deux signes en question sont là dans la version française reproduite dans l'ouvrage. Quoi qu'il en soit, les deux omissions confèrent à la version de Cohen un air moins structurel que les autres. Quant aux versions de Campos, elles sont manifestement les plus adaptatives côté ponctuation, car il a inséré des signes à des endroits stratégiques. Dans "Acompañamiento ", il a ajouté un deux-points au huitième vers, annonçant plus clairement que l'original les paroles rapportées du sujet lyrique: «y decir: soy yo». Étrangement, cela rapproche la version espagnole du poème autographe, où, comme nous l'avons vu plus haut, Saint-Denys Garneau avait mis un deux-points et même des guillemets pour encadrer les paroles rapportées. Cependant, comme Campos n'a probablement jamais eu accès au poème autographe, son intervention ressemble plus à une volonté de restituer ne serait-ce qu'un semblant de norme à la syntaxe garnélienne. Cette hypothèse se confirme à la lecture de sa version de "Cage d'oiseau », où Campos a ajouté des points d'interrogation à chacun des vers de la sixième strophe. Non seulement ces ajouts altèrent l'aspect dépouillé du poème, d'autant plus que l'espagnol requiert des points ouvrants et fermants, mais leur surabondance force carrément à lire les vers comme des questions et génère une atmosphère de panique, tandis que l'original ne fait que suggérer leur nature interrogative, l'absence de ponctuation instaurant pour sa part un ton neutre pointant vers la fonction rhétorique des questions. 


\subsection{Découpe des vers}

La majorité des traducteurs ont reproduit autant que faire se peut la découpe originale des vers, mais on constate, là aussi, des exceptions. $\mathrm{Si}$ les indentations à droite sont difficiles à calquer de façon parfaitement structurelle en raison des différences inévitables entre les mots français et espagnols, celles de gauche pourraient fournir une meilleure indication sur la façon dont cet aspect a été abordé en traduction. Par exemple, dans la version d' «Accompagnement» produite par Cohen, la longueur des blancs typographiques varie passablement, en français comme en espagnol. En fait, cela est vraisemblablement dû à l'original qu'elle a consulté. En effet, la source des poèmes de Garneau citée par Cohen est l'anthologie La poésie québécoise de Laurent Mailhot et Pierre Nepveu, qui présente exactement les mêmes blancs typographiques que ceux des versions de Cohen. Dans ce contexte, l'approche de Cohen est clairement structurelle à l'égard de la découpe des vers. Un autre cas d'exception est la version de Paraíso de Leal, qui ne comporte aucune indentation. Il est difficile de savoir si l'original auquel elle a eu accès est la source de ce contraste avec le poème de Garneau. Toutefois, l'effet créé est, lui, nettement adaptatif. D'une part, le poème ne comporte plus le profil aéré typiquement garnélien, ce qui accélère le rythme de lecture des vers. La manifestation graphique du «s'étiolant à ma gauche» a aussi complètement disparu, ce qui représente un appauvrissement stylistique. D'autre part, la nouvelle disposition entraîne un glissement de sens non négligeable dans la troisième strophe. En effet, le segment «por medio de malabarismos» ("par des jeux d'équilibre») ne peut plus faire partie de l'énumération entamée aux troisième et quatrième vers de la strophe, car il est devenu, sur le plan syntaxique, un complément obligé du groupe «Desplazamiento de átomos».

\section{Conclusion}

Nous avons proposé plus haut que faire de la traductologie comparée, ce serait étudier ces liens verticaux qui existent au sein d'une même langue cible ou entre diverses langues cibles, par l'étude simultanée de plusieurs traductions du même texte. C'est ce que nous avons fait ici en analysant les versions espagnoles de "Cage d'oiseau» et d'«Accompagnement» parues en Amérique latine. Dans un article au sujet du poème «Saules» traduit par John Glassco, Patricia Godbout, paraphrasant Barnstone, avance que «traduire le signifié sans se référer à son signifiant équivaut à un 
meurtre de l'art» (2009, p. 24). Alors, l'esthétique de Garneau a-telle survécu dans le prisme des traductions espagnoles de "Cage d'oiseau » et d' «Accompagnement » diffusées en Amérique latine?

Sur le plan sonore, notre analyse révèle que l'esthétique garnélienne est généralement sauve. D'une part, les versions de "Cage d'oiseau» importent toutes de façon structurelle les répétitions des substantifs centraux (cage, oiseau, os, mort), créant la même insistance qu'en français. Si les réitérations d'autres types de mots ne sont pas systématiques, l'effet itératif est le plus souvent préservé au moyen d'une approche fonctionnelle. D'autre part, dans "Accompagnement», la majorité reporte les segments anaphoriques et les répétitions lexicales de façon structurelle (Campos, Cohen, Paraíso de Leal) ou fonctionnelle (Aguinaga). Seules les versions de Fernández del Valle et Carvajal renferment assez de variations pour qu'on y voie les fruits d'une approche plus adaptative.

Sur le plan spatial, l'esthétique de Saint-Denys Garneau survit presque partout. D'abord, dans la plupart des publications, la mise en page semble surtout le fruit d'une approche fonctionnelle faisant un compromis entre les contraintes éditoriales et l'aspect graphique original, sauf chez Paraíso de Leal, où la disparition des vers tronqués produit un effet adaptatif, et chez Campos, où le saut de page dans "Cage d'oiseau» modifie l'enchaînement du poème. Mais les versions de Campos sont sans doute les plus adaptatives de toutes sur le plan spatial: ses ajouts de ponctuation sont si adaptatifs que l'on ne reconnaît plus l'architecture originale. À cet égard, presque tous les autres traducteurs ont reporté de façon structurelle la disposition originale des signes français.

Pour compléter cette étude de la poésie de Saint-Denys Garneau en espagnol, il serait souhaitable de procéder à une analyse du contenu des versions. À première vue, les traductions de Fernández del Valle et Carvajal paraissent les plus adaptatives, contenant bon nombre d'omissions et de glissements, voire de faux sens. Les versions d'Aguinaga contiennent aussi plusieurs éléments adaptatifs, mais les autres semblent plutôt fonctionnelles, mise à part la tendance structurelle manifeste chez Campos sur le plan syntaxique. Voilà des observations préliminaires qu'une étude plus poussée pourrait confirmer. 


\section{Références}

Ben Ari, Nitsa (1998). «The Ambivalent Case of Repetitions in Literary Translation. Avoiding Repetitions: A 'Universal' of Translation?» Meta, 43, 1, p. 1-11.

Berman, Antoine (1989). «La traduction et ses discours». Meta, 34, 4, p. 672-679.

Bérubé, Renald (1973). «Sur deux poèmes de Saint-Denys Garneau». Voix et images du pays, 6, 1, p. 91-102.

Blais, Jacques (1984). «Saint-Denys Garneau et le jeu des variantes». Études françaises, 20, 3, p. 29-50.

Blodgett, Edward D. (2012). «De la difficulté de traduire Saint-Denys Garneau en anglais », Études françaises, 48, 2, p. 111-119.

De Saint-Denys Garneau, Hector (1949). Poésies complètes. Montréal/ Paris, Fides.

De Saint-Denys Garneau, Hector (1971). Euvres. Montréal, Presses de l'Université de Montréal.

De Saint-Denys Garneau, Hector (1973). «Acompañamiento». Trad. Isabel Paraíso de Leal. Árbol de fuego, 66, p. 11.

De Saint-Denys Garneau, Hector (1996). «Acompañamiento »; «Jaula de pájaro ». In B. Pozier, dir. Poetas de Quebec, Trad. Lorenza Fernandez del Valle et Juan Carvajal. Trois-Rivières, Écrits des Forges; Mexico, Editorial Aldus/UNAM, p. 79-80.

De Saint-Denys Garneau, Hector (2003a). "Jaula de pájaro»; "Acompañamiento». In B. Pozier, dir. Latinos del norte: antología de poesía de Quebec. Trad. Marco Antonio Campos. Trois-Rivières, Écrits des Forges; Mexico, Fondo de Cultura Económica, p. 27 et 31.

De Saint-Denys Garneau, Hector (2003b), «Jaula de pájaro ». Pequeño fin del mundo. Trad. Luis Vicente de Aguinaga. Trois-Rivières, Écrits des Forges; Guadalajara, Filodecaballos Editores, p. 41-42.

De Saint-Denys Garneau, Hector (2006). «Acompañamiento»; «Jaula de pájaro». In S. Cohen, auteure et trad., La frontera de la lengua. Buenos Aires, Editorial Biblos, p. 41-42 et 74-75.

De Saint-Denys Garneau, Hector (2007). «Jaula de pájaro»; "Acompañamiento». In Todos y cada uno. Trad. Luis Vicente de Aguinaga. Trois-Rivières, Écrits des Forges; Guadalajara, Ediciones Arlequín, p. 117, 119 et 123.

Godbout, Patricia (2009). «L'expérience de l'origine: John Glassco, traducteur de Saint-Denys Garneau». Recherches sémiotiques/Semiotic Inquiry, 29, 2-3, p. 23-31.

Haeck, Philippe (1973). «Naissance de la poésie moderne au Québec». Études françaises, 9, 2, p. 95-113. 
Jones, D.G. (2004). « Hommage». In P. Bélanger, dir. Saint-Denys Garneau: la clef de lumière. Montréal, Éditions du Noroît (Chemins de traverse), p. 11-14.

Lemaire, Michel (1994). «Métrique et prosaïsme dans la poésie de SaintDenys Garneau». Voix et Images, 20, 1, p. 73-84.

Mainguy, Thomas (2012). «Saint-Denys Garneau: le jeu de l'ironie». Études françaises, 48, 2, p. 29-50.

Major, Jean-Louis (1972). «Saint-Denys Garneau et la poésie». Études françaises, 8, 2, p. 176-195.

Melançon, Robert (2004). «Notes de relecture de Saint-Denys Garneau». In P. Bélanger, dir. Saint-Denys Garneau: la clef de lumière. Montréal, Éditions du Noroît (Chemins de traverse), p. 51-61.

Nepveu, Pierre (1984). «La prose du poème». Études françaises, 20, 3, p. 15-27.

Riffaterre, Michael (1983). «On the Prose Poem's Formal Features». In M. A. Caws et H. Riffaterre, dir. The Prose Poem in France: Theory and Practice. New York, Columbia University Press, p. 117-132.

Ryan, Thomas D. (2003). «The Textual Presence of the Translator: A Comparative Analysis of F. R. Scott's and John Glassco's Translations of Saint-Denys Gameau ». Mémoire de maitrise, Université Concordia [Montréal]. Inédit.

Smoje, Dujka (1982). «Lorsque le verbe se fait musique: Saint-Denys Garneau». Études littéraires, 15, 1, p. 69-95.

Stratford, Madeleine (2011). «Un poème de Pizarnik sous toutes ses coutures: vers une nouvelle méthode d'analyse des traductions poétiques». TTR, 24, 1, p. 143-176.

Vigneault, Robert (1973). Saint-Denys Garneau à travers Regards et jeux dans l'espace. Montréal, Presses de l'Université de Montréal.

Zito, Marina (2004). «L'architecture de Regards et jeux dans l'espace». In P. Bélanger, dir. Saint-Denys Garneau: la clef de lumière. Montréal, Éditions du Noroît (Chemins de traverse), p. 107-123.

Madeleine Stratford Université du Québec en Outaouais Département d'études langagières 283, boul. Alexandre-Taché, bur. F-1052 Gatineau (Québec) J8X 3X7 CANADA madeleine.stratford@uqo.ca 


\section{Annexe I. «Cage d'oiseau " ${ }^{5}$ et ses traductions}

\section{CAGE D'OISEAU}

1 Je suis une cage d'oiseau

2 Une cage d'os

3 Avec un oiseau

4 Loiseau dans sa cage d'os [1971: Loiseau dans ma cage d'os]

5 C'est la mort qui fait son nid

6 Lorsque rien n'arrive

7 On entend froisser ses ailes

8 Et quand on a ri beaucoup

9 Si l'on cesse tout à coup

10 On l'entend qui roucoule

11 Au fond

12 Comme un grelot

13 C'est un oiseau tenu captif

14 La mort dans ma cage d'os

[Saut de page en 1949 et en 1971]

15 Voudrait-il pas s'envoler

16 Est-ce vous qui le retiendrez

17 Est-ce moi

18 Qu'est-ce que c'est

19 Il ne pourra s'en aller

20 Qu'après avoir tout mangé

21 Mon cœur

22 La source du sang

23 Avec la vie dedans

24 Il aura mon âme au bec.

5. Sources: De Saint-Denys Garneau (1949, p. 96-97) et De Saint-Denys Garneau (1971, p. 33-34). 


\begin{tabular}{|c|c|}
\hline $\begin{array}{c}\text { Lorenza Fernández del Valle et } \\
\text { Juan Carvajal (1996) }\end{array}$ & Marco Antonio Campos (2003) \\
\hline JAULA DE PÁJARO & JAULA DE PÁJARO \\
\hline $\begin{array}{l}\text { Soy la jaula de un pájaro } \\
\text { Una jaula de hueso } \\
\text { Con un pájaro }\end{array}$ & $\begin{array}{l}\text { Soy una jaula de pájaro } \\
\text { Una jaula de huesos } \\
\text { Con un pájaro }\end{array}$ \\
\hline $\begin{array}{l}\text { El pájaro en su jaula de hueso } \\
\text { Es la muerte que hace su nido }\end{array}$ & $\begin{array}{l}\text { El pájaro en mi jaula de huesos } \\
\text { Es la muerte que hace su nido }\end{array}$ \\
\hline $\begin{array}{l}\text { Cuando nada sucede } \\
\text { Se oyen frotar sus alas }\end{array}$ & $\begin{array}{l}\text { Cuando nada aparece } \\
\text { Se oye frotar sus alas }\end{array}$ \\
\hline $\begin{array}{l}\text { Y cuando se ha reído en exceso } \\
\text { Si se para de pronto } \\
\text { Se oye gorgear } \\
\text { Al fondo } \\
\text { Como un cascabel }\end{array}$ & $\begin{array}{l}\text { Y cuando se ha reído mucho } \\
\text { Si uno cesa de repente } \\
\text { Se le oye zurear } \\
\text { En el fondo } \\
\text { Como un cascabel }\end{array}$ \\
\hline $\begin{array}{l}\text { Es un pájaro cautivo } \\
\text { La muerte en mi jaula de hueso }\end{array}$ & $\begin{array}{l}\text { Es un pájaro cautivo } \\
\text { La muerte en mi jaula de huesos }\end{array}$ \\
\hline $\begin{array}{l}\text { Quisiera alzar el vuelo } \\
\text { Y eres tú quien lo retienes } \\
\text { Y soy yo } \\
\text { Lo que es } \\
\text { [Saut de page] }\end{array}$ & $\begin{array}{l}\text { ¿No quisiera emprender vuelo? } \\
\text { ¿Ustedes lo retendrían? } \\
\text { ¿Seré yo? } \\
\text { ¿Qué es? }\end{array}$ \\
\hline $\begin{array}{l}\text { No podrá irse } \\
\text { Sino después de devorar } \\
\text { Mi corazón } \\
\text { La fuente de la sangre } \\
\text { Con la vida adentro } \\
\text { Llevará mi alma en su pico. }\end{array}$ & $\begin{array}{l}\text { El no podrá partir } \\
\text { Sino después de haber comido todo } \\
\text { [Saut de page] } \\
\text { Mi corazón } \\
\text { La fuente de la sangre } \\
\text { Con la vida dentro } \\
\text { Tendrá mi alma en el pico. }\end{array}$ \\
\hline
\end{tabular}




\begin{tabular}{|c|c|}
\hline Sara Cohen (2006) & $\begin{array}{l}\text { Luis Vicente de Aguinaga } \\
(2007)\end{array}$ \\
\hline $\begin{array}{l}\text { Jaula de pájaro } \\
\text { Soy una jaula de pájaro } \\
\text { Una jaula de hueso } \\
\text { Con un pájaro } \\
\text { El pájaro en su jaula de hueso } \\
\text { Es la muerte que hace su nido } \\
\text { Cuando nada ocurre } \\
\text { Se oyen sus alas arrugar } \\
\text { [Saut de page] } \\
\text { Y cuando reímos mucho } \\
\text { Al detenernos de pronto } \\
\text { Lo escuchamos gorjear } \\
\text { Al fondo } \\
\text { Como un cascabel } \\
\text { Es un pájaro cautivo } \\
\text { La muerte en mi jaula de hueso } \\
\text { No querrá alzar vuelo } \\
\text { Es que acaso ustedes lo retendrían } \\
\text { Acaso soy yo } \\
\text { Qué es } \\
\text { No podrá irse } \\
\text { Sino después de haber comido todo } \\
\text { Mi corazón } \\
\text { La fuente de la sangre } \\
\text { Con la vida adentro } \\
\text { Tendrá mi alma en su pico. }\end{array}$ & $\begin{array}{l}\text { [blanc typographique de } 4 \mathrm{~cm} \text { ] } \\
\text { Soy una jaula de pájaro } \\
\text { Una jaula de huesos } \\
\text { Con un pájaro } \\
\text { El pájaro en mi jaula de huesos } \\
\text { Es la muerte al hacer su nido } \\
\text { Cuando no pasa nada } \\
\text { Se oyen sus alas al rozarse } \\
\text { Y cuando nos reímos mucho } \\
\text { Si nos callamos de golpe } \\
\text { La oímos arrullar } \\
\text { Al fondo } \\
\text { Como un cascabel } \\
\text { Es un pájaro cautivo } \\
\text { La muerte en mi jaula de huesos } \\
\text { Querría tal vez echarse al vuelo } \\
\text { No serás tú quien la detenga } \\
\text { No seré yo } \\
\text { Qué es } \\
\text { [Saut de page] } \\
\text { No podrá irse } \\
\text { Antes de habérselo comido todo } \\
\text { Mi corazón } \\
\text { El manantial de la sangre } \\
\text { Y adentro la vida } \\
\text { Tendrá mi alma en el pico. }\end{array}$ \\
\hline
\end{tabular}




\section{Annexe II. «Accompagnement ${ }^{6}$ et ses traductions}

\section{ACCOMPAGNEMENT}

1 Je marche à côté d'une joie

$2 \quad$ D'une joie qui n'est pas à moi

$3 \quad$ D'une joie à moi que je ne puis pas prendre

$4 \quad$ Je marche à côté de moi en joie

5 J'entends mon pas en joie qui marche à côté de moi

$6 \quad$ Mais je ne puis changer de place sur le trottoir

$7 \quad$ Je ne puis pas mettre mes pieds dans ces pas-là

$8 \quad$ et dire voilà c'est moi

9 Je me contente pour le moment de cette compagnie

10 Mais je machine en secret des échanges

11 Par toutes sortes d'opérations, des alchimies,

12 Par des transfusions de sang

13 Des déménagements d'atomes

$14 \quad$ par des jeux d'équilibre

15 Afin qu'un jour, transposé,

16 Je sois porté par la danse de ces pas de joie

17 Avec le bruit décroissant de mon pas à côté de moi

18 Avec la perte de mon pas perdu

19 s'étiolant à ma gauche

20 Sous les pieds d'un étranger

qui prend une rue transversale

6. Sources: De Saint-Denys Garneau (1949, p. 101) et De Saint-Denys Garneau (1971, p. 34). Dans l'édition de 1949, le titre est en majuscules, sur une autre page, et le poème est en italique. Dans l'édition de 1971, seul le titre est en italique, et il ne porte qu'une majuscule initiale. 


\section{Isabel Paraíso de Leal (1973)}

\section{ACOMPAÑAMIENTO ${ }^{7}$}

Camino al lado de una alegría

De una alegría que no es mía

De una alegría que no puedo alcanzar

Camino a mi lado en alegría

Oigo mi paso en alegría que camina a mi lado

Pero no puedo cambiar de lugar en la acera

No puedo poner mis pies en esos pasos y decir ya está soy yo

Me contento por ahora con esta compañía

Pero maquino en secreto intercambios

Por toda clase de operaciones, de alquimias,

Por transfusiones de sangre

Desplazamientos de átomos por medio de malabarismos

Para que un día, transformado,

Me lleve la danza de esos pasos de alegría

Con el ruido decreciente de mi paso a mi lado

Con la pérdida de mi paso marchitándose a mi izquierda

Bajo los pies de un desconocido que toma una calle transversal.

7. Le titre est présenté dans le coin supérieur droit de la page. 


\section{Lorenza Fernández del Valle et Juan Carvajal (1996)}

\section{ACOMPAÑAMIENTO}

Voy al lado de una alegría

De una alegría que no es mía

De una alegría que no puedo asir

Camino alegre al lado mío

Oigo a mi paso alegre que marcha al lado mío

Pero no puedo poner mis pies en esos pasos

$\mathrm{y}$ decir que soy yo

Me alegro de momento con esta compañía

Pero maquino cambios en secreto

Para toda clase de operaciones, alquimias,

Por medio de sanguíneas transfusiones

Descomposiciones de átomos

$$
\text { en juegos de equilibrio }
$$

Con el fin de que un día, transpuesto,

Salga con la danza de este pas de joie

Con el ruido decreciente de mi paso a mi lado

Con la pérdida de mis pasos perdidos

marchitándose a mi izquierda

Bajo los pies de un extranjero

que toma una calle transversal. 


\title{
Marco Antonio Campos (2003)
}

\author{
ACOMPAÑAMIENTO
}

Yo ando al lado de un goce

De un goce que no es mío

De un goce en mí que no puedo tomar

Yo ando al lado de mí gozoso

Oigo mi paso gozoso que anda al lado mío

Pero no puedo cambiar de sitio sobre la acera

No puedo poner mis pies en esos pasos

y decir: soy yo

Me contento por el momento con esta compañía

Pero maquino secretamente unos intercambios

Por toda suerte de operaciones, de alquimias,

Por transfusiones de sangre

Mudanzas de átomos

$$
\text { por juegos de equilibrio }
$$

A fin de que un día, transpuesto,

Sea llevado por el baile de esos pasos de goce

Con el ruido disminuido de mi paso al lado mío

Con la pérdida de mi paso perdido

debilitándose a mi izquierda

Bajo los pies de un extraño

que toma una calle transversal 


\section{Sara Cohen (2006)}

\section{Acompañamiento}

Camino al lado de una alegría

De una alegría que no es mía

De una alegría mía que no puedo tomar

Camino a mi lado en alegría

Escucho mi paso en alegría que camina a mi lado

Pero no puedo cambiar de lugar en la vereda

No puedo poner mis pies en esos pasos

y decir he aquí soy yo

[Saut de page]

Me contento por el momento con esta compañía

Pero tramo en secreto intercambios

Para toda suerte de operaciones, alquimias

Para transfusiones de sangre

Mudanzas de átomos

$$
\text { para juegos de equilibrio }
$$

A fin de que un día, traspuesto,

Sea llevado por la danza de esos pasos de alegría

Con el ruido decreciente de mi paso a mi lado

Con la pérdida de mi paso perdido marchitándose a mi izquierda

Bajo los pies de un extranjero

que toma una calle transversal 


\section{Luis Vicente de Aguinaga (2007)}

\section{Acompañamiento [sur la page précédente]}

\section{CAMINO AL PAREJO DE UN GOZO}

De un gozo que no me pertenece

De un gozo mí que no puedo asir

Camino junto a mi gozando

Oigo mi paso andar junto a mi gozando Pero no puedo cambiar de sitio en la banqueta No puedo poner mis pies en aquellos pasos diciendo mírenme soy yo

Me conformo por lo pronto con esa compañia Pero en secreto voy urdiendo intercambios Por toda clase de operaciones, de alquimias, Por transfusiones de sangre

Mudanzas de átomos

$$
\text { juegos de equilibrio }
$$

Para que un día, transportado,

Me lleve la danza con esos pasos de gozo

Con el ruido decreciente de mi paso a mi lado

Con la pérdida de mi paso perdido marchitándose a mi izquierda

Bajo los pies de un extraño que toma una calle transversal. 